\title{
Editorial
}

\section{Nutrigenomics and Cancer Therapy}

\author{
Jing X. Kang \\ Massachusetts General Hospital and Harvard Medical School, Boston, Mass., USA
}

As an increasingly prevalent disease and a leading cause of death, cancer is a major threat to human health. Although cancer research has provided us with a better understanding of cancer biology, we still face numerous challenges in cancer treatment and prevention. Current therapies are largely limited to surgery, radiation therapy, and chemotherapy, which remain unsatisfactory. In particular, there are many problems in chemotherapy, such as low response rate, poor specificity, drug resistance, and severe side effects. Thus, we still have much to do in order to improve the current situation. It is our priority to identify and develop alternative treatment options that can increase efficacy, reduce side effects, and improve quality of life for cancer patients. In this context, nutrigenomics is emerging as a field that holds great promise for this endeavor because of its capability to modulate cancer metabolism and tumorigenesis through nutritional intervention.

Cancer metabolism has gained unprecedented attention due to its involvement in every stage of cancer development. Cancer cells are characterized by an altered metabolism to sustain their rapid growth. One well-recognized alteration is the reprogramming of cellular energy production, known as the Warburg effect: unlike normal cells that primarily utilize oxidative phosphorylation, cancer cells predominantly produce energy by a high rate of glycolysis followed by lactic acid fermentation, and thus require a large supply of glucose [1]. Recent studies have revealed that changes in gene expression, such as phosphofructokinase 1 [2] and pyruvate kinase type M2 [3], are implicated in this altered pathway. Cancer cells also exhibit an increased production of certain metabolites that serve as building blocks to meet proliferation requirements. In particular, lipid synthesis is enhanced in cancer cells by the upregulation of fatty acid-metabolizing enzymes, such as fatty acid synthase [4], stearoyl-CoA desaturase-1 [5], and delta-6 desaturase [6]. Furthermore, the production of factors that support the tumor microenvironment is augmented in cancer cells. For example, one well-known metabolic biomarker of cancer is the overexpression of cyclooxygenase-2, which is responsible for converting omega- 6 arachidonic acid into tumor-promoting eicosanoids [7]. It is clear that nutrient metabolism is critical for cancer biology, and thus modulation of the metabolic pathways would have profound effects on cancer development.

Certain nutrients have been shown to be capable of modulating gene expression related to cancer hallmarks, such as inflammation, angiogenesis, and proliferation, through multiple mechanisms. For example, polyunsaturated fatty acids (PUFA) can influence gene expression 
in at least three ways: (1) PUFA can be incorporated into the cell membrane and thereby modify its chemical and physical properties, affecting many membrane-associated functional proteins (such as ion channels and receptors) that signal gene expression; (2) when metabolized, PUFA form bioactive lipid mediators (such as eicosanoids) that bind to receptors or associated proteins to trigger gene expression, and (3) PUFA and their metabolites can interact directly with a number of key transcriptional factors (such as PPARs, NFK $\beta$, and SREBP). Through these pathways, omega- 6 and omega-3 PUFA have been shown to differentially affect gene expression related to inflammation (e.g. TNF $\alpha$, IL-1 $\beta$, IL-6), angiogenesis (e.g. VEGF, PDGF, IGF-1, MMP-2), and proliferation (e.g. cyclin, p53, Wnt, PTEN) and can thereby modulate tumorigenesis [8, 9]. Furthermore, other food components, such as curcumin, epigallocatechin gallate, vitamin D, and certain phytochemicals, have also demonstrated anticancer effects through their impact on the expression of genes involved in these cancer-related processes. The promising effects of various nutrients on cancer-related gene expression provide a compelling basis for utilizing nutrients to modulate cancer metabolism and tumorigenesis.

The crucial role of nutrigenomics in the field of cancer therapy is apparent. By elucidating the network of nutrient-gene interactions related to cancer, we can ultimately synthesize this information into integrated metabolic interventions for cancer therapy. As these nutritional interventions can target multiple mechanisms, they could prove to be more effective than conventional therapies - in addition to being safer, more cost-effective, and more accessible for cancer patients. However, to implement nutrigenomics for cancer therapy, much translational research is still to be done. It is particularly important to develop clinically effective dietary protocols and supplement formulas for specific conditions as well as biomarkers to identify utility criteria and monitor efficacy.

Nutrigenomics therefore offers a novel approach to cancer management; conversely, cancer therapy is a critical field for the practice of nutrigenomics.

\section{References}

1 Warburg 0: On the origin of cancer cells. Science 1956;123:309-314.

- 2 Yi W, Clark PM, Mason DE, Keenan MC, Hill C, Goddard WA 3rd, Peters EC, Driggers EM, Hsieh-Wilson LC: Phosphofructokinase 1 glycosylation regulates cell growth and metabolism. Science 2012;337:975-980.

- 3 Luo W, Semenza GL: Emerging roles of PKM2 in cell metabolism and cancer progression. Trends Endocrinol Metab 2012;23:560-566.

- 4 Flavin R, Peluso S, Nguyen PL, Loda M: Fatty acid synthase as a potential therapeutic target in cancer. Future Oncol 2010;6:551-562.

5 Igal RA: Stearoyl-CoA desaturase-1: a novel key player in the mechanisms of cell proliferation, programmed cell death and transformation to cancer. Carcinogenesis 2010;31:1509-1515.

- 6 He C, Qu X, Wan J, Rong R, Huang L, Cai C, Zhou K, Gu Y, Qian SY, Kang JX: Inhibiting delta-6 desaturase activity suppresses tumor growth in mice. PLoS One 2012;7:e47567.

- 7 Greenhough A, Smartt HJ, Moore AE, Roberts HR, Williams AC, Paraskeva C, Kaidi A: The COX-2/PGE2 pathway: key roles in the hallmarks of cancer and adaptation to the tumour microenvironment. Carcinogenesis 2009; 30:377-386.

$\checkmark 8$ Kang JX, Liu A: The role of the tissue omega-6/omega-3 fatty acid ratio in regulating tumor angiogenesis. Cancer Metastasis Rev 2013;32:201-210.

9 Schmitz G, Ecker J: The opposing effects of n-3 and n-6 fatty acids. Prog Lipid Res 2008;47:147-155. 